\title{
Using Neural Network for forecasting in the Financial Sector
}

\author{
Barkhatov N.A. ${ }^{1}$, Revunov S.E. ${ }^{2},{ }^{*}$, Smirnova Zh.V. ${ }^{3}$, Cherney O.T. ${ }^{4}$, Katkova O.V. ${ }^{5}$ \\ ${ }^{1}$ Minin Nizhny Novgorod State Pedagogical University (Minin University), Nizhny Novgorod, Russian Federation \\ barkhatov@mail.ru \\ ${ }^{2}$ Minin Nizhny Novgorod State Pedagogical University (Minin University), Nizhny Novgorod, Russian Federation \\ revunovse@mail.ru \\ ${ }^{3}$ Minin Nizhny Novgorod State Pedagogical University (Minin University), Nizhny Novgorod, Russian Federation \\ smirnova@mail.ru \\ ${ }^{4}$ Minin Nizhny Novgorod State Pedagogical University (Minin University), Nizhny Novgorod, Russian Federation \\ cherney@mail.ru \\ ${ }^{5}$ Minin Nizhny Novgorod State Pedagogical University (Minin University), Nizhny Novgorod, Russian Federation \\ katkova@mail.ru
}

\begin{abstract}
Most areas of human activity, including the economy, require constant improvement. Every year, the volume of information and the speed of its change are rapidly increasing. Processing and managing so much human intelligence is inefficient, and using traditional computing becomes a time-consuming process. Therefore, modern information technologies come to the rescue. Today, various intelligent methods are widely used for data analysis, in particular, neural networks.

In order for an enterprise to function more effectively, many statistical methods and models are created, as well as specialized software. However, most methods lack Multi-linearity, it is possible to describe most processions and uniqueness of the stationary solution in systems equations, which makes it not accurate enough. In such cases, the use of neural networks as a method of modeling economic processes is Crucial. The purpose of this study was to highlight the concept of a piece neural network and the principles of its functioning. To investigate the use of neural networks as a method of forecasting and modeling economic refinancing processes, as well as to highlight the main types of software for working with neural networks.
\end{abstract}

Key words : Neural Network, Forecasting, Bank sector.

\section{INTRODUCTION}

Modeling of economic processes can be carried out using both traditional mathematical methods and modern methods, which include neural network technologies $[2,3]$. Neural networks allow us to solve problems that traditional methods cannot solve. they are able to solve problems based on incomplete, noisy, distorted information. An important advantage of neural networks for processing data sets is a significant increase in the speed of the process in comparison with traditional mathematical methods, the ability to train a neural network with reference samples, as well as changing the network topology (selection of input parameters that guarantee the highest accuracy of the model), based on the requirements of the problem to be solved. The concept of a neural network came from biology, where it is defined as a network consisting of biological neurons connected and functionally United in the nervous system. Although the neural network is a simplified model of the human brain, it is quite successfully used in solving a variety of tasks. Interest in the use of artificial neural networks in the economy is growing every day. They have proven themselves in solving many difficult financial and economic problems. Artificial neural networks are an indispensable tool for high-quality processing of huge data flows, without which it is very difficult, sometimes impossible, to adequately assess the market situation and make the right decision. All this indicates the need for further study, development and implementation of artificial neural networks in practice.

\section{MATERIALS AND METHODS}

It is advisable to use neural networks for solving poorly formed problems (requiring time-consuming calculations). These recognitions include:

1. Forecasting. This is the first class of economic problems that can be solved using artificial neural networks. It is their ability to generalize and detect hidden dependencies within network elements that allows them to cope with such tasks. examples are:

- forecasting the level of demand for a new product or service;

- forecasting sales volumes;

- predicting customer behavior;

- analysis of the firm's reliability and determination of the probability of its bankruptcy;

- predicting changes in the value of shares in a certain period of time; 
- forecasting the feasibility of implementing innovative projects and their economic efficiency;

- assessment of the client's solvency and the risk of granting him a loan.

2. the next type of problems that can be solved using artificial neural networks is the classification of objects of economic analysis. For example, the classification of clients by the degree of risk of providing them with a loan. Having considered the main types of economic problems that can be solved with the help of artificial neural networks, we can turn to the world practice, and see how some companies use neural technologies in their activities.

A striking example of the successful use of artificial neural methods can be the financial Corporation Citicorp, which uses a large specialized neurocomputer to analyze and predict short-term exchange rates. The combined accuracy of forecasts made by the neural me-network exceeded the results of experienced brokers of the Corporation. Another firm that has implemented market analysis using neural network technologies is Richard Borst, which trades in non-comission. Since the beginning of the use of the neuropacket, the company's turnover in New York and Moscow increased by 6\% [4].

An artificial neural network is a system consisting of many simple computational elements (neurons) that are connected in a certain way. The most common are multi-layer networks, in which neurons form layers. The layer, in turn, is a set of neurons that receive information from other neurons of the network in each clock cycle, that is, they are connected to the inputs of other neurons, so the signal from one element is transmitted to another. After determining the number of layers and the number of elements in each of them, the network needs to be trained $[5,6]$, that is, to determine values for the weights and dimensions of the network that would minimize the error of the forecast issued by the network. The error for a specific network configuration is determined by running through the network of all available observations and comparing the output values that are actually output with the desired (target) values. In essence, the learning process is a fitting of the model that is implemented by the network to the available training data. A typical example of how a neural network works is shown in figure 1 .

The input layer is intended simply for entering the values of input variables. Each of the input and output neurons is connected to all the elements of the previous. One could consider networks in which neurons are connected only from the neurons of the previous layer; however, for most cases it is better to give preference to networks with a complete system of connections.

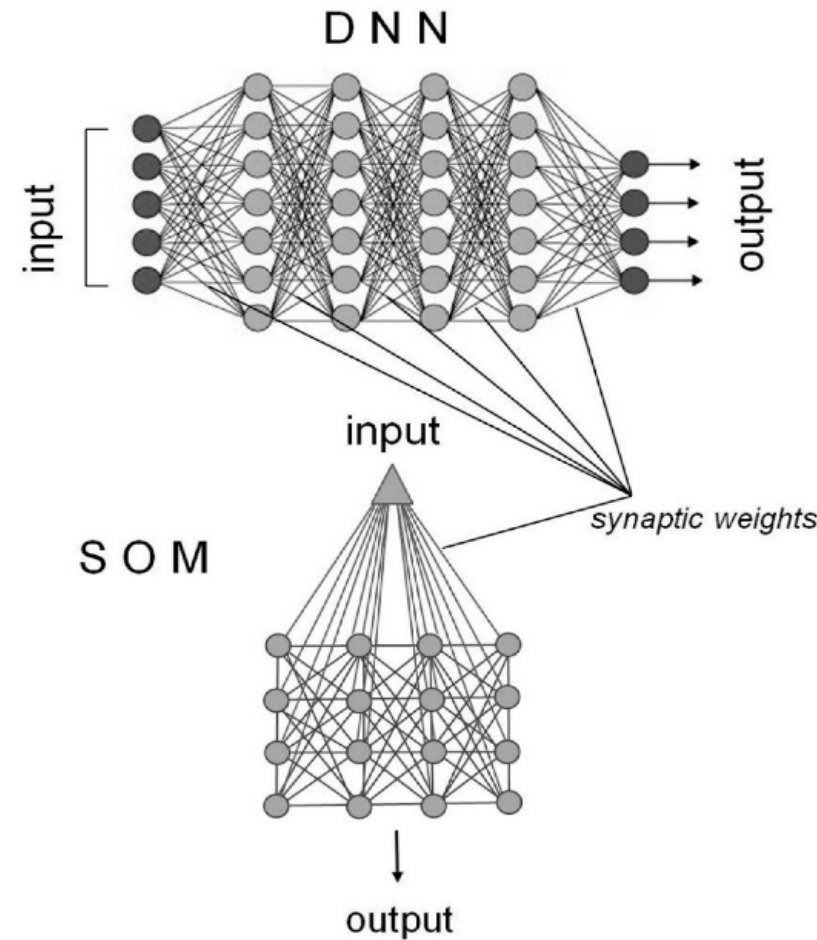

Figure 1: Neural network diagram currently there are several dozen structures of neural me-dir.

Since all artificial neural networks are based on the concept of neurons,connections, and transfer functions, there are similarities between different structures of neural networks. Most of the differences depends on the different training practices. For the learning process, it is necessary to have a model of the external environment in which the neural network functions-the necessary information for solving the problem. You also need to determine how to modify the network's weight parameters. Algo-learning rhythm refers to a procedure that uses learning rules to adjust weights. There are three General paradigms of learning: "with a teacher", "without a teacher" (self-learning), and mixed [7].To train a neural network is to tell it what we expect from it.

A neural network can learn with or without a teacher. After repeated presentation of examples, the weights of the neural network are stabilized, and the neural network gives correct answers to all (or almost all) applications from the database. In this case, it is said that "the neural network has studied all the examples", "the neural network is trained", or "the neural network is trained ". In software implementations, you can see that the error value (the sum of the error squares for all outputs) gradually decreases during the learning process.

When the error value reaches zero or an acceptable low level, the training is stopped, and the resulting neural network is considered trained and ready to use the new data. One of the most significant areas of application of neural networks in the financial sphere is forecasting on the stock market.

Standard methods that do not use neural networks are based on a rigid fixed set of "rules of the game", which eventually lose their relevance due to trading conditions on the stock exchange. In addition, systems of this type are 
too slow for situations that require the trader (bidder) to make decisions in a timely manner. Thus, the use of neural networks is a fairly powerful forecasting method that allows you to reproduce a fairly complex dependence. Neural networks for stock market forecasting have the following advantages:

- Easy to use, since neural networks are trained on prik-frets. The neural network user selects representative data, and then runs a training algorithm that automatically perceives the data structure.

- Neural networks are attractive from an intuitive point of view, because they are based on a primitive biological model of nervous systems. In the future, the development of such neurobiological models may lead to the creation of actual computers [8].

- Prediction of financial time series is a necessary element of any investment activity. The very idea of investing in order to generate income in the future is based on the idea of predicting the future.

The forecast of financial time series is the main activity of the entire investment industry - all exchanges and OTC systems of securities trading [9].

So, for a high-quality forecast, it is necessary to use high-quality prepared data, as well as a neuropack with greater functionality. A large number of specialized programs are designed to work with neural networks, some of which are more universal, while others are highly specialized.

Let's briefly look at some of the programs that are used:

1. Matlab-desktop laboratory for mathematical calculations, design of electrical circuits and modeling of complex systems. It has a built-in programming language and a very rich set of tools for neural networks-Anfis Editor (training, creation, training, and graphical interface), a command interface for programming networks, and Tool - for a more subtle network configuration. Suitable for the initial work of Forex market forecasting, [10] as well as in Fig. 2.the results of neural network modeling of the PFTS index based on data for the first 20 days of 2015 are shown. Actual data is indicated in black, and forecast data is indicated in gray [11].

The Elman neural network is used for modeling in the Matlabpackage[9]. This network allows you to remember your previous actions and implement learning tasks that unfold over time, which is relevant for predicting hours of memory problems.

2. Statistica is a fairly powerful software that is used to search and analyze data and identify statistical patterns. In this package, working with neural networks is presented in the STATISTICA Neural Networks module (abbreviated as ST Neural Networks, the StatSoft neural network package), which is an implementation of the entire set of neural network methods for data analysis [12].

3. NeuroShell Day Trader is a neural network system that takes into account the specific needs of traders, although it is easy to use, the program is quite specialized, it is suitable for trading, but in its essence it is very close to the black box [9].

When building a neural network for trading on the exchange market, a number of problems arise, the same as when creating a trading strategy without using neural networks. Since the market changes all the time, it is necessary to determine which period on the chart is used for training the neural network. The answer is the bottom before that, there may be problems here: if an unsuccessful period is selected, For example, with high volatility caused by a number of unpredictable and possibly non-recurring events in the future, this will lead to an error in the accuracy of the forecast [13].

4. BrainMaker-this package is intended for solving problems for which formal methods and algorithms have not yet been found, and the input data is incomplete, distorted, and contradictory. These tasks include exchange and financial forecasting, crisis modeling, pattern recognition, and many others. BrainMaker is one of the first packages and market leaders. At first, it was commissioned by the military. The package was adapted for business applications in the 90th year and was awarded the prestigious PC Magazine award "Best software product of the year" [15-23].

Since then, the package has won various competitions, survived 20,000 installations (which is quite a lot for a specialized package) and is now the most popular neuropack in the United States [24, 25].

\section{CONCLUSION}

The use of neural networks as a method of modeling economic processes is relevant in the context of the problem when processing and managing a large amount of data by human intelligence is inefficient, and the use of traditional calculations becomes a labor-intensive process. The use of neural networks is a powerful predictive method that allows us to reproduce fairly complex dependencies. It solves many urgent problems in the sphere of economy and Finance. One of the most important areas of application of neural networks in the financial sphere is forecasting on the stock market. The use of neural networks is a fairly powerful forecasting method that allows us to predict fairly complex dependencies. The variety of software for implementing artificial networks gives economists-analysts a wide range of choices, which will be carried out depending on the tasks set, as well as the area of research.

\section{REFERENCES}

1. A.V. Guglielmi, V.A. Troitskaya. Geomagnetic pulsations and magnetosphere diagnostics. Moscow: Ed. Nauka, 1973. 208 p. (in Russian)

2. N.A. Barkhatov. Ultra low-frequency wave attenuation in the transition region // Geomagnetism and aeronomy, 1982. Vol. 22. pp. 819-823 (in Russian)

3. M.Bitskaya, (2018). Ecologically adaptive receptions control the number of pests in the ecosystems of transformed at the forest reclamation. World Ecology Journal, 8(2), 1-10. https://doi.org/https://doi.org/10.25726/NM.2018.2. 2.001

4. A. Wawrzaszek, W.M. Macek. Observation of the 
multifractal spectrum in solar wind turbulence by Ulysses at high latitudes // J. Geophys. Res., 2010. Vol. 115, A07104. doi:10.1029/2009JA0151763.

5. J.A. Tessein, C.W. Smith, B.J. Vasquez, R.M. Skoug. Turbulence associated with corotating interaction regions at $\mathbf{1}$ AU: Inertial and dissipation range magnetic field spectra // $\mathrm{J}$. Geophys. Res., 2011. Vol. 116, A10104. doi:10.1029/2011JA016647

6. K. Steed, C.J. Owen, P. Démoulin, S. Dasso. Investigating the observational signatures of magnetic cloud substructure // J. Geophys. Res., 2011. Vol.116, A01106, doi:10.1029/2010JA015940.

7. I. Daubechies. TenWavelet Lectures. Izhevsk: Ed. NIC «Regular and chaotic dynamics», 2001. 464 p. (in Russian)

8. N.M. Astafieva. Wavelet analysis: theory and application examples // Advances in the Physical Sciences, 1996. Vol. 166, No 11. pp. 1145-1170

9. N.A. Barkhatov, V.G. Vorob'ev, S.E. Revunov, O.I. Yagodkina. Effect of solar dynamics parameters on the formation of substorm activity // Geomagn. Aeron., 2017. Vol. 57, Iss. 3. pp 251-256, https://doi.org/10.1134/S0016793217030021

10. Yu.V. Manakova, K.A. Pekhteleva, N.A. Barkhatov, S.E. Revunov. Space-time analysis of perturbations of the Pc4-5 range during periods of magnetic storms by the correlation-skeleton method // Vestnik of Minin University. 2016. no. 1. pp. 1-6.

11. N.A. Barkhatov. Development of methods for predicting the geomagnetic state of the magnetosphere based on the search for fundamental laws of solar-terrestrial relationships // Vestnik of Minin University. 2013. no. 2. pp. 1-11.

12. N.A. Barkhatov, S.E. Revunov, A.B. Vinogradov. The classification algorithm for MHD wavelet-skeleton spectral patterns of geoeffective plasma flows in the solar wind // Vestnik of Minin University. 2014. no. 3. pp. 1-6.

13. S.E. Revunov, S.I. Kuznetsov, O.M. Barkhatova, E.A. Revunova. The problem of connection of observer's mind with quantum-mechanical description of physical reality // Vestnik of Minin University. 2019. Vol. 7, no. 3. P. 14

14. N.A. Barkhatov, V.G. Vorobjev, S.E. Revunov, O.M. Barkhatova, and O.I. YagodkinaSubstormActivity and Orientation of the Front of a Shock Wave of an Interplanetary Magnetic Cloud // Geomagnetism and Aeronomy, 2019, Vol. 59, No. 4, pp. 398-406. C Pleiades Publishing, Ltd., 2019.

15. N.A. Barkhatov, E.A. Revunova, O.M. Barkhatova, R.V. Romanov, S.E. Revunov. Studying the relationship of localization parameters of solar sources of magnetic clouds with their characteristics and substorm activity // Solar-Terrestrial Physics. 2019. Vol. 5. Iss. 3. P. 59-67. DOI: 10.12737/stp-53201907.
16. N.A. Barkhatov, S.E. Revunov, M.V. Mukhina, M.L. Gruzdeva, O.T. Cherney and Z.V. Smirnova. Establishing the orientation of shock wave plane of solar wind magnetic cloud for conclusions about the level of auroralsubstorm activity. JP Journal of Heat and Mass Transfer. Volume 17, Issue 1, Pages 195 - 202 (June 2019) http://dx.doi.org/10.17654/HM017010195, 2019

17. N.A. Barkhatov, S.E. Revunov, V.G. Vorobjev, and O.I. Yagodkina, Studying the relationship between high-latitude geomagnetic activity and parameters of interplanetary magnetic clouds with the use of artificial neural networks // Geomagn. Aeron., 2018, vol. 58, no. 2, pp. 147-153. https://doi.org/10.7868/S0016794018020013

18. N.A. Barkhatov, D.S. Dolgova, and E.A. Revunova, Dependence of the geomagnetic activity on the structure of magnetic clouds // Geomagn. Aeron., 2019, vol. 59, no. 1, pp. 16-26.

19. O.M. Barkhatova, N.V. Kosolapova, N.A. Barkhatov, S.E. Revunov. Synchronization of geomagnetic and ionospheric disturbances over Kazan station // Solar-Terrestrial Physics, 2017. Vol. 3. No. 4. pp. 58-66, doi: 10.12737/stp-34201706

20. S.V. Klyuev, S.N. Bratanovskiy, S.V. Trukhanov, H.A. Manukyan. Strengthening of concrete structures with composite based on carbon fiber // Journal of Computational and Theoretical Nanoscience. 2019. V.16. №7. P. 2810 - 2814.

21. N. Ivanisova\& L. Kurinskaya. 2019. Biogeochemical activity of park plants as an indicator of stability of wood plants. World Ecology Journal, 9(1), 40-54. https://doi.org/https://doi.org/10.25726/NM.2019.20 .18 .003

22. Gribust. 2019. Environmental elements for revitalization of entomophages in the forest plantations of the arid zone. World Ecology Journal, 9(1), 55-69. https://doi.org/https://doi.org/10.25726/NM.2019.86 .67 .004

23. S.V. Klyuev, S.N. Bratanovskiy, S.V. Trukhanov, H.A. Manukyan. Strengthening of concrete structures with composite based on carbon fiber // Journal of Computational and Theoretical Nanoscience. 2019. V.16. №7. P. 2810 - 2814.

24. Dwidienawati, D., Abdinagoro, S. B., Gandasari, D., \&Nadira. (2019). Young customers' perception on influencer endorsement, customer review and E-tailing channel. International Journal of Advanced Trends in Computer Science and Engineering, 8(6), 3369-3374. https://doi.org/10.30534/ijatcse/2019/110862019

25. Ebad, S. A., \& Ahmed, M. (2019). A comparison of functionality-based packaging using ga and adaptive knn clustering as two approaches to package software. International Journal of Advanced Trends in Computer Science and Engineering, 8(1.4 S1), 428-433. https://doi.org/10.30534/ijatcse/2019/6781.42019 